\title{
Micro-scale surface-patterning influences biofilm formation
}

\author{
Guimel M. Kappell \\ Department of Biology \\ The University of Texas at Arlington \\ Arlington Texas, 76019, USA \\ James P. Grover \\ Department of Biology \\ The University of Texas at Arlington \\ Arlington Texas, 76019, USA \\ Thomas H. Chrzanowski* \\ Department of Biology \\ The University of Texas at Arlington \\ PO Box 19498, Arlington Texas, 76019, USA \\ Tel: 8172722404 \\ Fax: 8172722855 \\ E-mail: chrz@uta.edu
}

Keywords: indwelling medical devices, infections, surface attachment.

Abbreviations: EPS: extra-polymeric substances
SiE: silicone elastomer
TSB: tryptic soy broth

The formation of biofilms on indwelling/implanted medical devices is a common problem. One of the approaches used to prevent biofilm formation on medical devices is to inhibit bacterial attachment by modification of the synthetic polymers used to fabricate the device. In this work, we assessed how micro-scale features (patterns) imprinted onto the surface of silicone elastomer similar to that used for medical applications influenced biofilm formation by Staphylococcus aureus, Staphylococcus epidermidis, and Pseudomonas aeruginosa. Patterns were transferred from a multipatterned oxidized silicon-wafer master-template to silicone elastomer. Features consisted of bars, squares, and circles each extending $0.51 \mu \mathrm{m}$ above the surface. Feature sizes ranged between 1.78 and $22.25 \mu \mathrm{m}$. Distances separating features ranged between 0.26 and $17.35 \mu \mathrm{m}$. Bacterial biofilm formation on discs cut from imprinted silicone elastomer was assessed by direct microscopic observation and quantified as the surface area covered by biofilm. Unpatterned silicone elastomer served as a control. Several of the micro-scale patterns imprinted into the silicone elastomer significantly reduced biofilm formation by each bacterium and interrupted biofilm continuity. Although there were differences in detail among strains, bacteria tended to attach in the area between features more than to the surface of the feature itself.

Bacterial cells attached to the surfaces of indwelling medical devices were discovered almost three decades ago (Costerton et al. 1987; Donlan, 2001; Costerton et al. 2003). Bacteria attaching to surfaces of such devices create a biofilm by synthesizing extracellular matrix polymers also known as glycocalyx or extra polymeric substance (EPS). These polymers are responsible for mediating adhesion and giving structure to the microbial community enclosed within (Branda et al. 2005). Since reported, a wide variety of medical devices have been found to harbor bacteria biofilms including sutures, shunts, contact lenses, and catheters (Costerton et al. 1999). The formation of biofilm on such devices by pathogenic bacteria is considered a virulence factor (Hall-Stoodley et al. 2004; Hall-Stoodley and Stoodley, 2005) and varies depending on the type of organism and type of biomaterial (Waldvogel and Bisno, 2000). Eradication of these infections usually requires complete removal of the prosthetic device and its adherent biofilm, which is sometimes impossible or undesirable. Long-term antibiotic suppression is employed in these situations but it is typically only marginally effective. Since biofilms protect the bacteria from host immune defense mechanisms and antibiotic drugs, infections are often impossible to control especially when the device remains in place (Donlan, 2001; Costerton et al. 2003; Hall-Stoodley and Stoodley, 2005).

Since biofilm formation on synthetic polymers leads to infections that are impossible to eradicate without device removal, prevention of biofilm formation on synthetic

*Corresponding author 


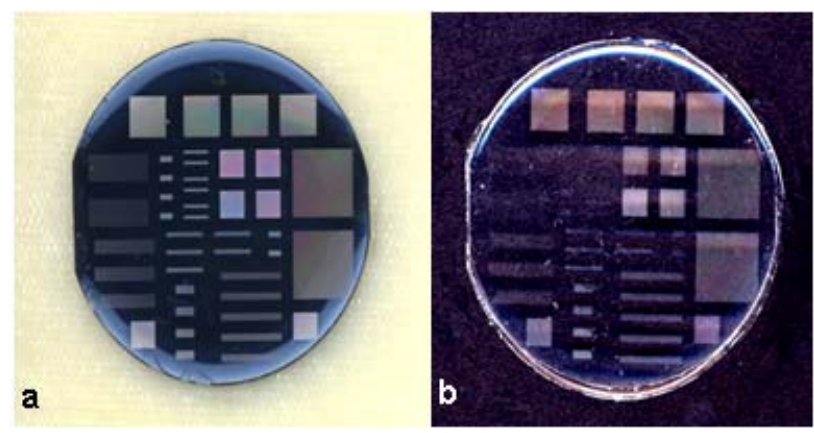

Figure 1. a) Master-template: oxidized-silicon wafer etched with various patterns by reactive ion etching. The diameter of the oxidized-silicon wafer is $5 \mathrm{~cm}$. b). Patterns transferred from a master-template to silicone elastomer.

polymers is an alternative step in the prevention of these infections. Two strategies for prevention and control of such biofilm formation have been described: 1) modification of polymers using physicochemical methods to obtain anti-adhesive material; and 2) incorporation of antimicrobial substances into current polymer biomaterials to obtain devices resistant to bacterial colonization. There has been limited success with the first strategy (Jansen and Peters, 1991; Lopez-Lopez et al. 1991; Jansen and Kohnen, 1995), and the second strategy exposes colonizing organisms to antibiotics and can lead to rapid development of drug resistance. The ideal material for medical implants should permit the binding of host defense cells but resist the adherence of blood compounds and bacteria (Gristina, 1987; Götz, 2002).

In this work, we used an in vitro proof-of-concept approach to explore a third strategy to reduce biofilm formation on a silicon elastomer similar to that used for a variety of medical applications. We describe how micro-scale patterns imprinted into the surface of the silicon elastomer may alter biofilm formation by three bacteria commonly isolated from, or associated with, infections related to indwelling medical devices (Staphylococcus aureus, Staphylococcus epidermidis, and Pseudomonas aeruginosa). We assessed biofilm formation on seven different patterns and compared them to biofilm formation on unpatterned surfaces.

\section{MATERIALS AND METHODS}

\section{Overview}

Biofilm formation on silicone elastomer ( $\mathrm{SiE}$ ) with surfaces containing different geometric patterns was compared to biofilm formation on unpatterned $\mathrm{SiE}$ surfaces. Geometric patterns were transferred from a multi-patterned oxidized silicon-wafer master-template to SiE. Biofilm on discs cut from patterned and unpatterned $\mathrm{SiE}$ was quantified using a combination of epifluorescence microscopy and image analysis.

\section{Master-templates}

A collection of patterns were etched into oxidized siliconwafers using a combination of UV-sensitive photoresist, lithographic mask, and mask aligner/exposure system. The photoresist pattern was transferred into the oxide layer by reactive ion etching (Figure 1). The multi-patterned templates were used as a master to imprint the different patterns into $\mathrm{SiE}$ (Figure 1) (Wilkerson et al. 2001). The patterns are characterized by feature (the portion projecting from the surface), feature height (distance the feature projects above the surface) and the periodicity (distance between features measured perpendicular to the feature) (Figure 2). Seven patterns were selected for study; three in which the feature was bars, three in which the feature was squares, and one in which the feature was circles (Table 1, Figure 2). Unpatterned surfaces served as controls.

\section{Transfers}

A $10: 1$ mix of Sylgard ${ }^{\circledR} 184$ Silicone Elastomer Base and Sylgard ${ }^{\circledR} 184$ Silicone Curing Agent (Dow Corning) was vortexed until uniform (2-3 min), transferred to a $50 \mathrm{~mL}$

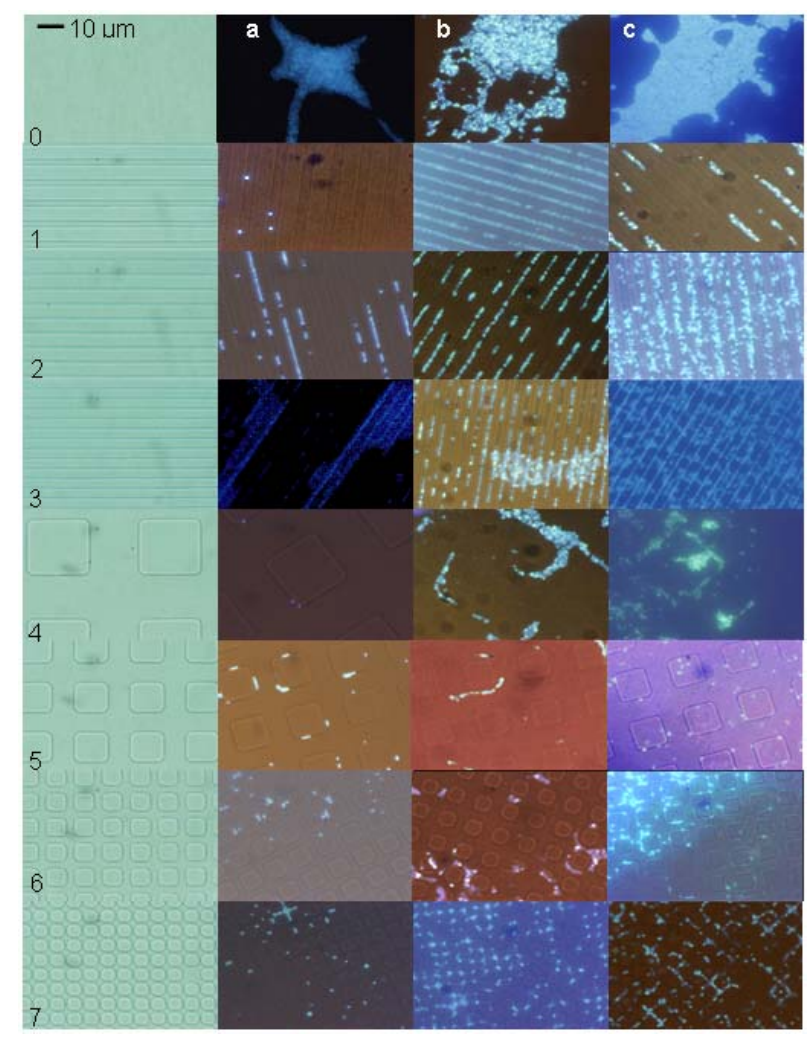

Figure 2. Features imprinted into silicone elastomer and growth of $S$. aureus (a), $S$. epidermidis (b), and $P$. aeruginosa (c) on imprinted silicone elastomer after $20 \mathrm{hrs}$. Patterns (bright field images) are identified in the left-most column and growth of individual organisms is shown in adjacent columns (combination epifluorescence and bright field images). Controls ( 0 ) were smooth surfaces and patterns consisted of bars $(1,2$, and 3$)$, squares $(4,5$, and 6$)$ and circles (7). Dimensions of features and spaces between features are given in Table 1. 
round flask (Corning 4320-B), and degassed (290 mm Hg, 15-20 min). A plastic ring was placed around a mastertemplate to contain the liquid $\mathrm{SiE}$ after it was poured over the template. Air bubbles were removed with an inoculating needle. The $\mathrm{SiE}$ was cured at $100^{\circ} \mathrm{C}$ for $1 \mathrm{hr}$ after which it was removed from the ring and template. Measurements of features and periodicity were determined for patterns imprinted into SiE using bright field microscopy and image analysis (Simple PCI, Compix, Inc) of photographs (Olympus DP80 digital camera) captured at $412 \mathrm{X}$. Feature height was measured using confocal microscopy (Zeiss LSM 5 Pascal) at $100 \mathrm{X}$.

\section{Discs containing patterns}

Discs ( $1 \mathrm{~cm} \mathrm{dia)} \mathrm{containing} \mathrm{a} \mathrm{single} \mathrm{pattern} \mathrm{were} \mathrm{cut} \mathrm{from}$ transfers (\#7 cork-borer) and adhered to the bottom inside surface of a glass Petri dish using a drop of SiE mix (cured as above). Dishes were subsequently sterilized by autoclaving. Each Petri dish contained four discs, three different patterns and a control, which were placed close to the edge of the dish at compass points. For consistency, bar-patterns were placed with the pattern parallel to the direction of the culture media flow (described below).

\section{Organisms and growth conditions}

Staphylococcus aureus (ATCC 6538), S. epidermidis (ATCC 35983) and P. aeruginosa (ATCC BAA-47) were obtained from the American Type Culture Collection and maintained at room temperature on Tryptic Soy Agar (Difco). Prior to use in experiments (see below), bacteria were grown overnight $\left(37^{\circ} \mathrm{C}\right.$, shaken) in Tryptic Soy Broth
(TSB).

Twenty $\mathrm{mL}$ of $0.01 \%$ TSB was aseptically added to Petri dishes containing discs and inoculated with $0.5 \mathrm{~mL}$ of an overnight culture of $S$. aureus, $S$. epidermidis or $P$. aeruginosa. The dishes were incubated at $37^{\circ} \mathrm{C}$ for $5 \mathrm{hrs}$ on an orbital table (Barnstead/LabLine 1314, 70 RPM) after which the plates were allowed to incubate statically for an additional $15 \mathrm{hrs}$.

\section{Quantitative assessment of biofilm formation}

Each disc was aseptically removed from its Petri dish, placed on a glass slide, and gently rinsed (2x) with distilled water to remove non-adherent cells remaining from the growth medium. Residual rinse water was carefully removed using a micropipette between rinses. Cell motion within $P$. aeruginosa biofilms interfered with image analysis; consequently, biofilms were fixed with one drop of $1 \%$ formaldehyde prior to rinsing. Adherent cells were stained with fluorochrome (4',6-diamidino-2-phenylindole) (Porter and Feig, 1980) for 2 min after which the stain was removed with a micropipette, one drop of immersion oil added, and biofilm visualized by epifluorescent microscopy (1250 X). Photographs (Olympus DP80 digital camera) of at least 15 randomly-selected fields were taken of each disc and the area covered by attached cells was quantified using image analysis software (Simple PCI, Compix, Inc). The field-of-view of each image was $3582 \mu \mathrm{m}^{2}$ and no visual field overlapped the edge of a disc.

In a separate set of experiments, S. epidermidis was inoculated into four-sets of three Petri dishes; one dish

Table 1. Characteristics of patterns imprinted into the surface of silicone elastomer (SiE). Feature is the width of the shape rising above the surface of the SiE. All features were $0.51 \pm 0.02 \mu \mathrm{m}^{\mathrm{a}}$ above the surface of the SiE. Periodicity is the space between features.

\begin{tabular}{|c|c|c|}
\hline Pattern & Feature - Size $(\boldsymbol{\mu m})^{\mathbf{b}}$ & Periodicity $(\boldsymbol{\mu m})$ \\
\hline 0 & None & \\
\hline 1 & Bar $-7.32 \pm 0.07$ & $2.73 \pm 0.09$ \\
\hline 2 & Bar $-5.00 \pm 0.04$ & $0.94 \pm 0.01$ \\
\hline 3 & Bar $-3.50 \pm 0.05$ & $0.46 \pm 0.03$ \\
\hline 4 & Square $-22.25 \pm 0.04$ & $17.35 \pm 0.07$ \\
\hline 5 & Square $-4.08 \pm 0.01$ & $2.52 \pm 0.01$ \\
\hline 6 & Square $-2.46 \pm 0.01$ & $0.92 \pm 0.01$ \\
\hline 7 & Circle $-1.78 \pm 0.01$ & $0.26 \pm 0.01$ \\
\hline
\end{tabular}

${ }^{a}$ Mean \pm SE, $n=22$

${ }^{\mathrm{b}}$ Mean $\pm \mathrm{SE}, \mathrm{n}=6$ for each feature 


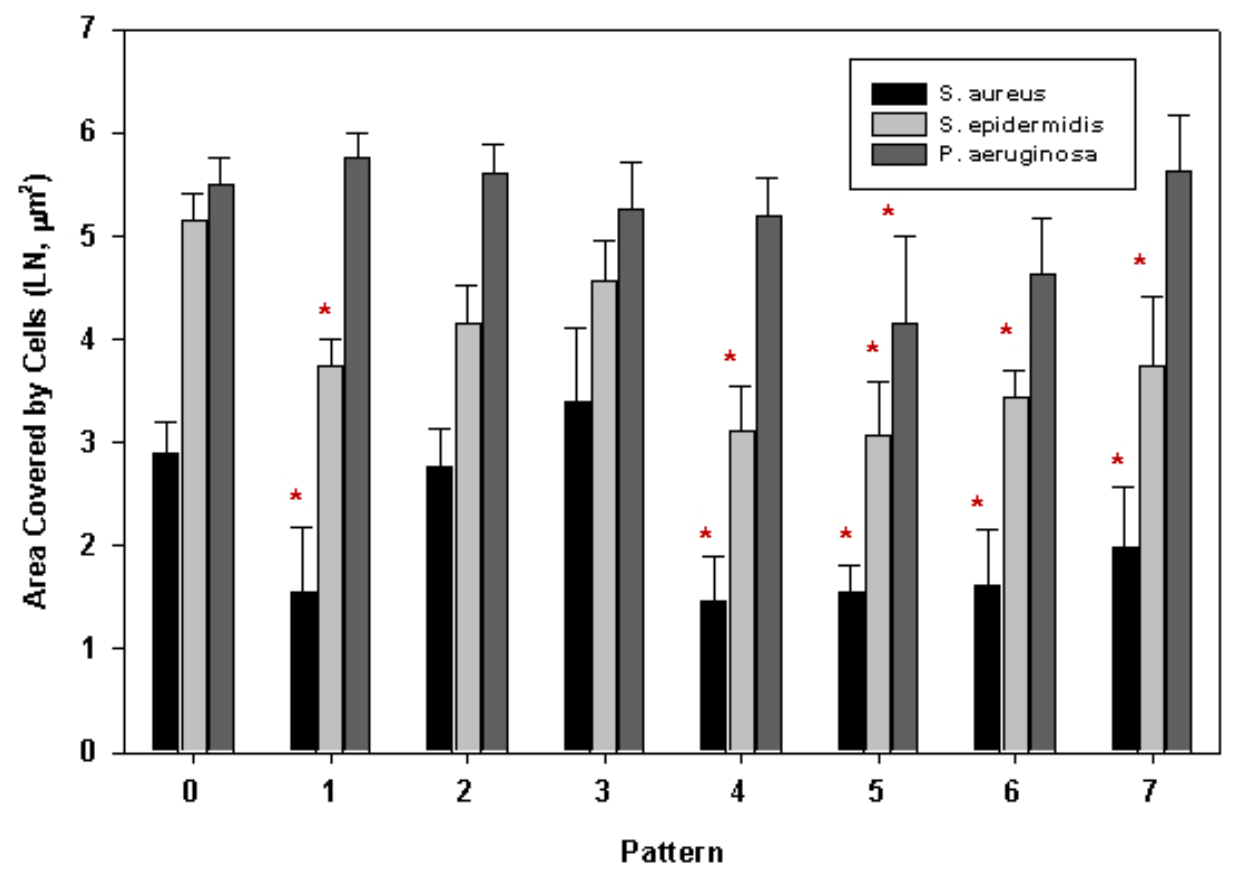

Figure 3. Area of silicone elastomer covered by biofilm. Patterns are identified in Table 1 and Figure 2. Values are the mean of measures obtained from 15 photographs of each of 6 discs. Error bars are the standard error of the mean $(n=6)$. Asterisks indicate that the area covered by biofilm was significantly different (ANOVA, $p<0.05$ ) than that found on smooth surfaces (controls, pattern 0 ).

containing 3 discs of pattern 4 (Table 1) and one control disc, one dish containing 3 discs of pattern 5 (Table 1) and one control disc, and one dish containing 4 control discs. All dishes were inoculated at the same time and one set of three dishes was removed after 48, 72, 96, and $120 \mathrm{hrs}$ of incubation. Biofilm formation was assessed as above.

\section{Statistical analyses}

Experiments were designed using 4 discs per Petri dish where 1 disc was always the control, 6 repetitions of each of the 7 patterns, and 14 repetitions of the control. The differences in biofilm formation among the patterns were determined using ANOVA for unbalanced incomplete blocks with post hoc comparisons performed using the Fisher least significant difference test (Statistica, StatSoft Inc.).

\section{RESULTS}

A sharp cork-borer produced discs of uniform diameter and free of rough edges. Discs varied in thickness between 1.5 and $3.0 \mathrm{~mm}$. Patterns were imprinted into $\mathrm{SiE}$ with very high reproducibility with low variability in feature size, periodicity, and height (Table 1). The use of a single growth medium in which all three types of bacteria grew well, allowed us to eliminate medium as a potential source of variability when assessing biofilm formation. Diluted medium was used to create a short period of logarithmic growth and to limit overall cell abundance.
Each type of bacterium formed dense multi-layer biofilms on unpatterned $\mathrm{SiE}$ (Figure 2, pattern 0); however, distinctly different growth patterns occurred when cells were grown on patterned surfaces. When grown on bar-type patterns (Figure 2, patterns 1-3) with long periodicity, bacteria attached between features and tended to grow in monolayers. Examples of this type of growth are shown in Figure 2, pattern 1. As the periodicity decreased, biofilms developed over the feature and began to bridge the gap separating the features. This type of growth may be seen for $P$. aeruginosa (Figure 2, patterns 2 and 3; and for S. aureus and S. epidermidis, pattern 3). When the feature was squares, $S$. aureus and $S$. epidermidis tended to grow between features no matter the periodicity (Figure 2, patterns 4-6); however $P$. aeruginosa tended to grow on the feature surface as well as between the features. $P$. aeruginosa biofilm grew over the features when the periodicity was small (Figure 2, pattern 6). Growth of each bacterium on $\mathrm{SiE}$ imprinted with circles, was similar to that occurring on bars with the greatest periodicity (Figure 2, compare pattern 7 to pattern 1 )

The area of each pattern covered by biofilm is summarized in Figure 3. Several patterns significantly reduced biofilm formation when compared to growth on unpatterned controls. Overall, 5 of the 7 patterns significantly $(p<0.05)$ reduced biofilm formation by $S$. aureus and by $S$. epidermidis, but only one was effective at significantly $(p<$ 0.05 ) reducing biofilm formation by $P$. aeruginosa. 
When compared to controls, patterns 4 and 5 were found to significantly reduce biofilm formation by both strains of Staphylococcus and pattern 5 was found to significantly reduce $P$. aeruginosa biofilm formation during $20 \mathrm{hrs}$ incubations. Consequently, these patterns were selected to assess their effectiveness in reducing biofilm formation during longer incubation periods (up to $120 \mathrm{hrs}$, Figure 4). The strain of $S$. epidermidis used in these studies consistently forms an abundant biofilm on $\mathrm{SiE}$ and was chosen to investigate longer-term biofilm development on patterns 4 and 5 . The biofilm formed when S. epidermidis was growing over pattern 4 was consistently reduced compared to that of biofilm formation on unpatterned $\mathrm{SiE}$ and significantly less $(p<0.05)$ than that growing on controls at 48 and $120 \mathrm{hrs}$. Biofilm formed on pattern 5 was significantly less than that growing on unpatterned $\mathrm{SiE}$ at 48 and 120 hrs but significantly greater than on unpatterned $\mathrm{SiE}$ at 72 and 96 hrs.

\section{DISCUSSION}

In the present work we altered the surface architecture of silicone elastomer similar to that used in medical devices and assessed the ability of three strains of bacteria to form biofilms on these textured surfaces. Among the microorganisms that are most frequently associated with biofilm derived infections are S. aureus, S. epidermidis, and $P$. aeruginosa. We chose these bacteria because of the frequency in which they (or strains thereof) occur in device associated infections but also because they differ in such characteristics as size, shape, motility, cell structure, and extracellular polysaccharide matrix (anionic (Sutherland, 2001) or cationic (Mack et al. 1996)). When growing under the conditions described above, $S$. aureus and $S$. epidermidis were $0.63 \pm 0.02$ and $0.86 \pm 0.03 \mu \mathrm{m}$ in diameter (mean $\pm \mathrm{SE}, \mathrm{n}=10$ ), respectively. Pseudomonas aeruginosa averaged $1.18 \pm 0.03 \mu \mathrm{m} \times 0.66 \pm 0.04 \mu \mathrm{m}(\mathrm{L} \times$ $\mathrm{W}$, mean $\pm \mathrm{SE}, \mathrm{n}=10$ ). Several $S$. aureus and $S$. epidermidis isolates produce cationic EPS matrix (polysaccharide intercellular adhesion) consisting mainly of 1, 6 - $\beta$ - $N$-acetylglucosamine. In contrast, anionic EPS for Pseudomonas species is formed by alginate, a linear polymer of 1, 4 - $\beta$-mannuronic acid and glucuronic acid (Ghannoum and O'Toole, 2004). Even though there are differences among the strains used in this study, there appears to be a general theme governing biofilm formation on the patterns imprinted on SiE: each of the strains tended to attach more often in the grooves between features than to the surface of the feature itself. Depending upon the periodicity, the surface feature also interrupted biofilm continuity.

While we note some general themes on how the bacteria tended to attach to $\mathrm{SiE}$, there were notable differences among the strains used in this work. Staphylococcus aureus formed biofilms covering equal or greater area than controls when growing on patterns 2 and 3 . These patterns were characterized by features (bars) having a small size and short periodicity. Consequently, S. aureus tended to form bridges that allowed it to overgrow the feature. Those patterns where the features were squares and circles $(4,5,6$ and 7), as well as those where the feature was bars with

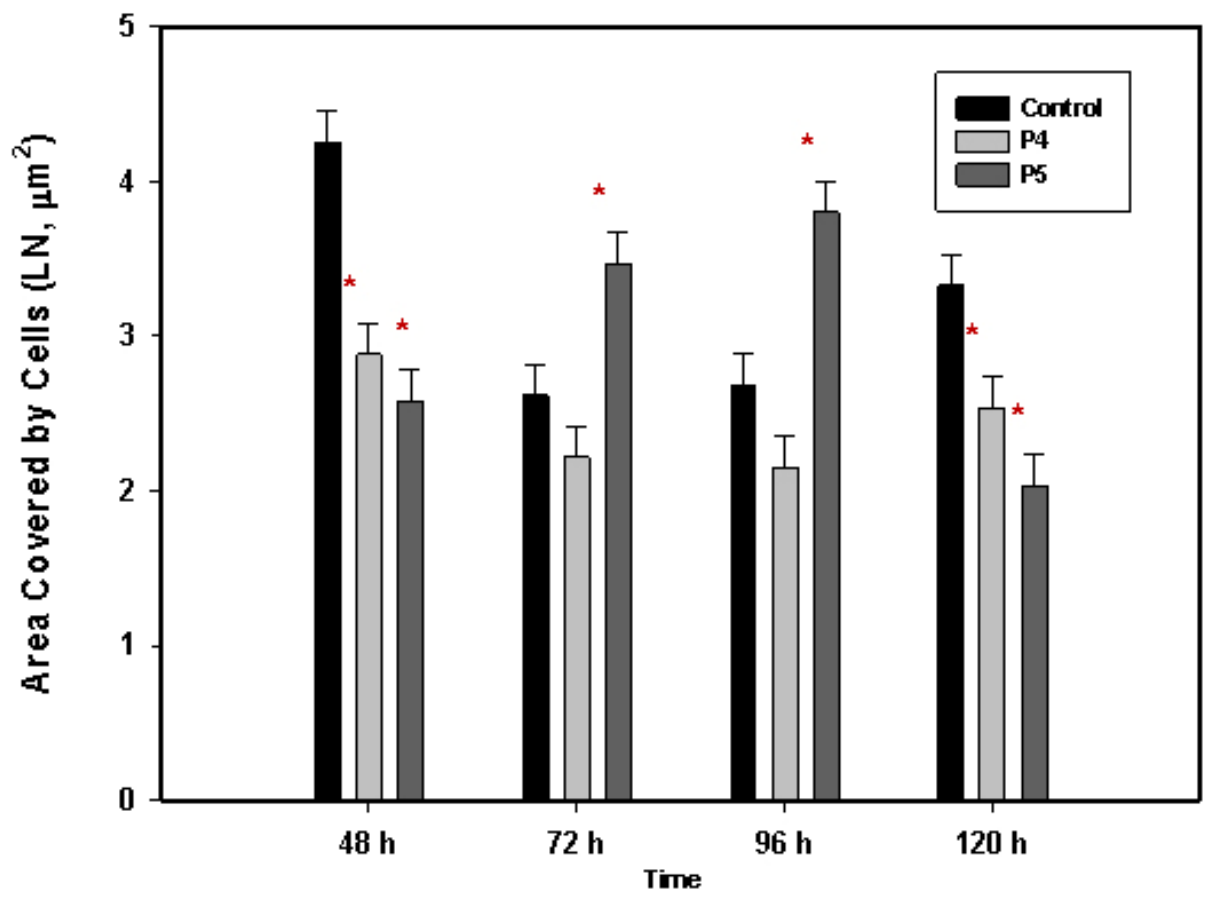

Figure 4. Area of silicone elastomer covered by $\mathbf{S}$. epidermidis biofilm at various time intervals. Values are the mean of measures obtained from 15 photographs of each of 3 discs. Error bars are the standard error of the mean $(n=3)$. Asterisks indicate that the area covered by biofilm was significantly different (ANOVA, $p<0.05$ ) than that found on smooth surfaces (controls, pattern 0 ). 
greater periodicity (pattern 1) seemed to effectively reduce early biofilm formation. Overall, S. aureus biofilms covered less area than did those of $S$. epidermidis or $P$. aeruginosa. Some have suggested (Waldvogel and Bisno, 2000; Götz, 2002) that S. aureus requires serum proteins to attach in vivo; these conditions were not met in this work and may account for sparse biofilm compared to that formed by $S$. epidermidis and P. aeruginosa. However, others have found greater variability in biofilm formation by staphylococci and this variability appears to be associated with the type of serum protein and, perhaps, the nature of the substrate (Herrmann et al. 1988), and the strain understudy. Even though we did not explore the influence of serum proteins on biofilm formation, it remains clear that micro-scale patterning of surfaces influenced how cells of the three strains attached to surfaces.

Similar to our finding with $S$. aureus, the area of surface covered by biofilm of $S$. epidermidis was greater when the features imprinted on $\mathrm{SiE}$ were closer to each other than when the features were more distant. The strain of $S$. epidermidis used in this work is known for abundant production of EPS and extensive biofilm formation; nevertheless, all patterns except patterns 2 and 3, significantly reduced biofilm formation when compared to unpatterned controls during short-term incubation while pattern 5 proved most effective at reducing biofilm formation during longer-term incubation.

Contrary to results obtained for staphylococci, $P$. aeruginosa biofilms on bar-patterned surfaces (patterns 1, 2 and 3) covered an area approximately equal to that growing on controls. Only one of the seven patterns (pattern 5) significantly reduced biofilm formation when compared to the control. This pattern was characterized by intermediatesized squares with intermediate periodicity.

Most of our initial conclusions based on short incubation times with $S$. epidermidis were unchanged after experiments were allowed to continue for much longer time periods; however, results were considerably more variable. Even though pattern 5 (squares with medium feature size and medium periodicity) was the pattern that worked best at reducing bacterial attachment in short-term experiments, pattern 4 seemed better than pattern 5 at reducing biofilm formation in longer incubations. It seems likely that some of the variability associated with longer-term incubations resulted from sloughing from, and subsequent re-growth of biofilm from either the control disks or from the treatment disks. Compare for example the loss of biofilm from control disks between 48 and $72 \mathrm{hrs}$ and the subsequent regrowth of biofilm by $120 \mathrm{hrs}$. Such a result was not anticipated by the original experimental design and clearly impacts our analyses.

The biofilms formed on surfaces were assessed by twodimensional measures (area). It is clear that most of the biofilms formed on patterned surfaces where the biofilm did not overgrow the feature were monolayers. However, in experiments where the biofilm did overgrow the feature, the biofilm was multi-layered. Similarly controls formed biofilms that were several cell-layers thick. Consequently, the impact of altering surface architecture on reducing biofilm formation is much greater for some patterns than the data suggest since the controls were multilayered but the experimental patterns produced largely monolayer biofilm. Additionally, features failing to reduce biofilm compared to controls may have actually produced significantly greater biofilm on some patterns. Clearly, three-dimensional quantification (e.g. confocal microscopy) should follow preliminary assessment of a given pattern for a given application.

The implications of this work are straightforward; altering surface architecture may delay the onset of biofilm formation by retarding development and disrupting continuity. Such patterning may find application in situations where wetted surfaces are contained in static frequently-contaminated environments. For example, patterning the base of a contact lens case or the tip of an eye-drop bottle may reduce biofilm development, especially over short-term use, and potentially decrease the incidence of ocular infections. Similarly, patterning the surface of a catheter may delay the onset of biofilm formation and allow it to remain in use for a longer period of time.

Cells found at surfaces of biofilms are generally thought to be more metabolically active and susceptible to antimicrobials and immune defenses than those imbedded with the biofilm matrix (Marshall, 1992; Donlan and Costerton, 2002; Fux et al. 2003). Micro-scale patterning of surfaces resulting in non-confluent biofilms should increase biofilm surface-area and expose more surface bacteria to antimicrobials and/or phagocytosis. Conceivably, nonconfluent biofilms should result in reduced quorum sensing signaling (O’Toole et al. 2000; Cámara et al. 2002; Yarwood et al. 2004; Parsek and Greenberg, 2005) and thus reduce the cascade of metabolic changes brought about by matrix development. Thus for long-term applications, patterning surfaces of certain types of implantable devices may extend the time the device may remain functional.

\section{ACKNOWLEDGMENTS}

The authors would like to express their thanks to Dr. R. Magnusson, University of Texas at Arlington, Dr. P. Priambodo, Universitas Indonesia, Dr. K. Pawlowski, University of Texas Southwestern Medical Center, and Debra Warwo, Resonant Sensors Incorporated, Arlington, Texas for creating the oxidized silicon-wafer mastertemplates, developing the original idea to explore their use in retarding biofilm development, and for advice on the manuscript. We would also like to thank Dr. Candace Mitchell, MedStudy Corporation, Colorado Springs, Colorado, for her advice on both the manuscript and the potential applications of the technology. The comments of 
an anonymous reviewer greatly improved the presentation of this information.

\section{REFERENCES}

BRANDA, Steven S.; VIK, Ashilid; FRIEDMAN, Lisa and KOLTER, Roberto. Biofilms: the matrix revisited. Trends in Microbiology, January 2005, vol. 13, no. 1, p. 20-26.

CÁMARA, Miguel; WILLIAMS, Paul and HARDMAN, Andrea. Controlling infection by tuning in and turning down the volume of bacterial small-talk. The Lancet Infectious Diseases, November 2002, vol. 2, no. 11, p. 667676.

COSTERTON, J.W.; CHENG, K.J.; GEESEY, G.G.; LADD, T.I.; NICKEL, J.C.; DASGUPTA, M. and MARRIE, T.J. Bacterial biofilms in nature and disease. Annual Review of Microbiology, October 1987, vol. 41, p. 435-464.

COSTERTON, J.W.; STEWART, P.S. and GREENBERG, E.P. Bacterial biofilms: a common cause of persistent infections. Science, May 1999, vol. 284, no. 5418, p. 13181322.

COSTERTON, J. William; VEEH, Richard; SHIRTLIFF, Mark; PASMORE, Mark; POST, Christopher and EHRLICH, Garth. The application of biofilm science to the study and control of chronic bacterial infections. The Journal of Clinical Investigation, November 2003, vol. 112 , no. 10 , p. 1466-1477.

DONLAN, Rodney M. Biofilms and device-associated infections. Emerging Infectious Diseases, March-April 2001, vol. 7, no. 2, p. 277-281.

DONLAN, Rodney M. and COSTERTON, J. William. Biofilms: survival mechanisms of clinically relevant microorganisms. Clinical Microbiology Reviews, April 2002, vol. 15 , no. 2, p. 167-193.

FUX, Christoph A.; STOODLEY, Paul; HALLSTOODLEY, Luanne and COSTERTON, J. William. Bacterial biofilms: a diagnostic and therapeutic challenge. Expert Review of Anti-infective Therapy, December 2003, vol. 1, no. 4, p. 667-683.

GHANNOUM, Mahmoud and O'TOOLE, George A. Microbial Biofilms. Washington, D.C.; ASM Press, 2004. 426 p. ISBN 1-55581-294-5.

GÖTZ, Friedrich. Staphylococcus and biofilms. Molecular Microbiology, March 2002, vol. 43, no. 6, p. 1367-1378.

GRISTINA, A.G. Biomaterial-centered infection: microbial adhesion versus tissue integration. Science, September 1987, vol. 237, no. 4822, p. 1588-1595.
HALL-STOODLEY, Luanne; COSTERTON, J. William and STOODLEY, Paul. Bacterial biofilms: from the natural environment to infectious diseases. Nature Reviews Microbiology, February 2004, vol. 2, no. 2, p. 95-108.

HALL-STOODLEY, Luanne and STOODLEY, Paul. Biofilm formation and dispersal and the transmission of human pathogens. Trends in Microbiology, January 2005, vol. 13, no. 1, p. 7-10.

HERRMANN, M.; VAUDEAUX, P.E.; PITTET, D.; AUCKENTHALER, R.; LEEW, P.D.; SCHUMACHERPERDREAU, F.; PETERS, G. and WALDVOGEL, F.A. Fibronectin, fibrinogen and laminin act as mediators of adherence of clinical staphylococcal isolates to foreign material. Journal of Infectious Diseases, October 1988, vol. 158, no. 4, p. 693-701.

JANSEN, B. and PETERS, G. Modern strategies in the prevention of polymer-associated infections. Journal of Hospital Infection, October 1991, vol. 19, no. 2, p. 83-88.

JANSEN, B. and KOHNEN, W. Prevention of biofilm formation by polymer modification. Journal of Industrial Microbiology and Biotechnology, October 1995, vol. 15, no. 4, p. 391-396.

LOPEZ-LOPEZ, G.; PASCUAL, A. and PEREA, E.J. Effect of plastic catheter material on bacterial adherence and viability. Journal of Medical Microbiology, June 1991, vol. 34 , no. 6 , p. 349-353.

MACK, Dietrich; FISHER, Werner; KROKOTSCH, Andreas; LEOPOLD, Klaus; HARTMANN, Rudolf; EGGE, Heinz and LANFS, Rainer. The intercellular adhesion involved in biofilms of Staphylococcus epidermidis is a linear $\beta$-1,6-linked glucosaminoglycan: purification and structure analysis. Journal of Bacteriology, January 1996, vol. 178, no. 1, p. 175-183.

MARSHALL, K.C. Biofilms: an overview of bacterial adhesion activity and control at surfaces. American Society for Microbiology News, April 1992, vol. 58, no. 4, p. 202207.

O'TOOLE, George; KAPLAN, Heidi B. and KOLTER, Roberto. Biofilm formation as microbial development. Annual Review of Microbiology, October 2000, vol. 54, p. 49-79.

PARSEK, M.R. and GREENBERG, E.P. Sociomicrobiology: the connections between quorum sensing and biofilms. Trends in Microbiology, January 2005, vol. 13, no. 1, p. 27-33.

PORTER, Karen G. and FEIG, Yvette S. The use of DAPI for identifying and counting aquatic microflora. Limnology and Oceanography, September 1980, vol. 25, no. 5, p. 943948. 
SUTHERLAND, Ian W. Biofilm exopolysaccharides: a strong and sticky framework. Microbiology, January 2001, vol. 147, no. 1, p. 3-9.

WALDVOGEL, Francis A. and BISNO, Alan L. Infections associated with indwelling medical devices. 3rd ed. Washington, D.C.; ASM Press, 2000. 470 p. ISBN 978-15581-177-8.

WILKERSON, Wade R; SEEGERT, Charles A.; FEINBERG, Adam W.; ZHAO, Lee C.; CALLOW, James A.; CALLOW, Maureen E. and BRENNAN, Anthony B. Bioadhesion studies on microtextured siloxane elastomers. Polymer Preprints, April 2001, vol. 42, no. 1, p. 147-148.

YARWOOD, Jeremy M.; BARTELS, Douglas J.; VOLPER, Esther M. and GREENBERG, E. Peter. Quorum sensing in Staphylococcus aureus biofilms. Journal of Bacteriology, March 2004, vol. 186, no. 6, p. 1838-1850. 